\title{
Lumen
}

Selected Proceedings from the Canadian Society for Eighteenth-Century Studies

\section{Le vocabulaire de la frontière : emprunts lexicaux amérindien/français et français/anglais au dix-huitième siècle}

\section{Peter W. Halford}

Volume 16, 1997

Freedom and Boundaries

Émancipation et frontières

URI : https://id.erudit.org/iderudit/1012442ar

DOI : https://doi.org/10.7202/1012442ar

Aller au sommaire du numéro

Éditeur(s)

Canadian Society for Eighteenth-Century Studies / Société canadienne d'étude du dix-huitième siècle

ISSN

1209-3696 (imprimé)

1927-8284 (numérique)

Découvrir la revue

Citer cet article

Halford, P. W. (1997). Le vocabulaire de la frontière : emprunts lexicaux amérindien/français et français/anglais au dix-huitième siècle. Lumen, 16 , 93-102. https://doi.org/10.7202/1012442ar 


\section{Le vocabulaire de la frontière: emprunts lexicaux amérindien/français et français/anglais au dix-huitième siècle}

Franchir une frontière géographique c'est parfois faire face à des réalités pour lesquelles la langue maternelle n'a pas de dénomination et de là, être obligé à nommer la nouveauté. C'est certainement le cas des explorateurs et colonisateurs français au moment de leur arrivée en Nouvelle-France aux dix-septième et dix-huitième siècles. Selon le témoignage des manuscrits du père Potier, ${ }^{1}$ des Jésuites, et des journaux de voyage de visiteurs tels que le baron de Lahontan, Chaussegros de Léry et le père Charlevoix, entre autres, après un siècle et demi de présence, les Canadiens avaient évolué deux stratégies d'adaptation lexicale qui se sont avérées productives. La première et la plus usitée était de puiser dans le fonds français et d'assigner à un signifiant pré-existant un nouveau signifié et de représenter la nouvelle réalité de cette manière. Ce procédé est à l'origine des sens canadiens de rouge-gorge pour la grive Turdus migratorius (cf. l'anglo-américain robin), chat ou chat sauvage pour le raton laveur, Procyon lytor et poisson blanc, pour le corégone, Coregonous clupeiformis, pour ne citer que trois exemples parmi les centaines qui en ont été répertoriées. Un deuxième procédé, celui qui nous intéresse ici, était d'emprunter à une langue usitée sur place un signifiant pour combler la lacune dans la langue maternelle.

Les emprunts aux langues amérindiennes par les Français et, plus tard, au français par les Britanniques, sont d'autant plus intéressants que les opinions des chercheurs sont variées. Robert A. Hall trouve que "surprisingly, despite the intensive contacts between French voyageurs and settlers and Algonquian or Iroquoian Indians in Canada, virtually no words have come into French from Amerindian sources except through eighteenth- or nineteenth-century English (e.g. mocassin, squaw, wigwam, scalper)» (159). Selon Claude Poirier, les contacts entre les populations indigènes et les Français au dix-huitième siècle étaient «assez peu importants sauf dans le monde des coureurs de bois» (66). La position de Marcel Juneau, dans son appréciation du manuscrit Potier, est un peu plus nuancée. Cet auteur signale que plusieurs mots relevés au Détroit 
par le père Potier «ne sont plus connus du québécois actuel» mais que leur présence même dans le manuscrit indique que «l'apport du substrat amérindien, faible dans le québécois d'aujourd'hui, était jadis bien plus important» (16).

Un examen des emprunts inventoriés par le père Potier en plein dix-huitième siècle révèle qu'un certain nombre ont été incorporés dans la francophonie mondiale et figurent dans des dictionnaires modernes $\mathrm{du}$ français général. Des vocables comme achigan, assimine, assiminier, atoca, babiche, carcajou, caribou, malachigan, manitou, maskinongé, ouaouaron, et pacane, pour n'en citer qu'une dizaine, se retrouvent dans des dictionnaires comme le Trésor de la langue française, Grand Robert et même le Petit Robert. On notera que la plupart de ces emprunts ont trait à la flore et à la faune.

D'autres vocables enregistrés par le père Potier ou par ses confrères missionnaires n'ont jamais franchi les pages des dictionnaires usuels du français général mais ont été des éléments importants dans le lexique de l'Amérique francophone, comme en témoignent les lexiques et glossaires depuis le père Potier au dix-huitième siècle et Jacques Viger au début du dix-neuvième siècle jusqu'au Glossaire $d u$ parler français au Canada et parfois même des éditions modernes comme le Dictionnaire des canadianismes de G. Dulong, le Dictionnaire Plus de Claude Poirier ainsi que le futur Trésor de la langue française au Québec. Parmi ces vocables, certains (comme apecia «jeune chevreuil» et killiou "sorte d'oiseau») ont trait à la faune, mais la plupart désignent des réalités quotidiennes. On trouve des mots comme apola «brochette de viande cuite au feu»; micoine «grosse cuiller en bois»; ouragan "plat en écorce» et sagamité «bouillie au blé d'Inde» entre autres, qui se rattachent à la cuisine amérindienne, ainsi que des vocables comme apichimon "marchepied, coussin, etc.», coutagan «couteau croche» et mitasse "guêtre» qui font partie de l'équipement du travail journalier. D'autres touchent à la vie des sociétés européenne et amérindienne en contact et mettent en relief les différences entre ces cultures. On trouve donc des exemples comme chichikoi «sorte de hochet», instrument toujours d'usage lors des conseils, des danses et des jongleries des autochtones; machicoté "cotron de sauvagesse», ${ }^{2}$ vêtement bien plus court que ceux des Européennes (v. Bonnefons 173); mattachiat "mélange de couleurs», que les autochtones appliquaient tant au corps qu'aux vêtements; panis «esclave américain». ${ }^{3}$ Enfin, signalons sakakoi: chez Potier, le mot signifie "crie de guerre» mais, à travers les siècles, on relève des variantes phonétiques et graphiques ainsi que des évolutions de sens. Vers la fin du siècle passé, Dunn consigne sasaqua «tapage» (169); au début de ce siècle, Clapin donne sacakoua et sasagua «... grand tapage, orgie infernale» ainsi que sasaqua et sasaqué «... bruit, hurlement, tapage» 
(287) et, plus tard, Glos consigne sassaqua «bruit, tapage, vacarme» (612b).

On note aussi des formes francisées et incorporées dans le système français par suffixation: mentionnons seulement quelques exemples répertoriés par Potier: attaronter "chanter la guerre», dont l'étymon semble être un radical huron atiron (v. Campeau 36); coutaganer «travailler avec le coutagan»; illinois "provenant du pays des Ilini ou Irini»; se mattachier "se barbouiller le visage» (attesté depuis le début du dixseptième siècle); micoinée "contenu d'une micoine»; panise «esclave amérindienne» et piroguée "contenu d'une pirogue».

Enfin, il y a les vocables qui n'ont été recensés que dans la région du Détroit et, parfois, dans les régions plus à l'Ouest, les vocables dont la diffusion semble être limitée à l'ancienne vie frontalière. ${ }^{4}$ Parmi les emprunts de cette catégorie, on trouve dans les documents Potier et dans d'autres documents ayant trait à la région (tels que les Relations des Jésuites et les recherches modernes de Hull, Johnson, Almazan, Halford et Bénéteau) des exemples comme agaga «farine bouilli avec du lard fricassé»; akokoine "perche qu'on penche pour y suspendre la chaudière»; aouapou "provisions»; endichon «appartement [c'est-à-dire, rayon, espace où ranger les affaires» (v. Campeau 117-118)] d'en haut dans une cabane sauvage»; escapia "valet de sauvage»; michilagué «appartement [v. endichon] d'en bas dans les cabanes sauvages»; okantican "grosse flotte aux deux bouts du maitre de rets»; ouindigo "poisson cannibale, chien de mer» et ouisseni «repas».

Le présent examen, si sommaire qu'il soit, indique clairement que l'importance des emprunts aux langues amérindiennes a été bien plus considérable que l'évaluation de Robert Hall ne nous laisserait croire, non seulement au niveau du français général mais aussi dans les variantes américaines et en particulier dans le parler de la frontière, le parler du Détroit et de l'intérieur de l'empire français en Amérique.

Devenus héritiers de ce vaste empire à la suite du Traité de Paris, les Britanniques qui sont venus explorer, traiter et s'établir dans l'ancienne Nouvelle-France, ont suivi les pistes, tant géographiques que linguistiques, établies par leurs précurseurs canadiens. Ceux-ci, s'étant déjà adaptés lexicalement à leur territoire et à ses réalités, fournissaient aux nouveaux-arrivés la terminologie qui manquaient dans leur anglais. En parcourant les mots d'origine française ou canadienne consignés dans le Dictionary of Canadianisms on Historical Principles, on remarque que les emprunts au substrat francophone sont non seulement nombreux mais qu'ils se sont parfois intégrés si solidement dans l'anglais nord-américain que le non-spécialiste ne soupçonne pas l'origine des vocables, comme c'est le cas de carry-all et de coulee. Par ailleurs, on est frappé par le nombre élevé de ces termes qui sont qualifiés de «désuet» ou de 
«historique» et, avec Juneau, on pourrait dire que «l'apport du substrat était jadis bien plus important».

Notre but n'est pas de présenter en détail les particularités des emprunts au français dans la période immédiatement après la Conquête mais plutôt d'esquisser en grandes lignes quelques-uns des aspects du processus tels que nous les trouvons dans les écrits des premiers explorateurs, commerçants et colons anglophones du dix-huitième siècle, en nous appuyant surtout sur les journaux des deux explorateurs du nom d'Alexander Henry et de John Askin. Ce dernier, commerçant à Michilimackinac et au Détroit devenu bilingue, ne mélange pas les deux langues: il écrit soit en français, soit en anglais. Alexander Henry I, explorateur et commerçant $n$ 'incorpore qu'une vingtaine de désignations françaises dans son vocabulaire mais parmi ces vocables on trouve la première attestation en anglais américain de praline. Qui plus est, en 1776, le mot a toujours le sens noté par Potier vingt-deux ans auparavant, c'est-à-dire «blé d'Inde gralé». Le journal d'Alexander Henry II, neveu du précédent, composé vingt ans plus tard, foisonne d'emprunts au français: nous en avons répertorié plus d'une centaine parmi lesquels il y a même quelques mots empruntés par les Canadiens aux langues amérindiennes.

Dans son texte, l'auteur a incorporé - comme s'il s'agissait d'un mot anglais - bien des vocables ayant trait à la flore et à la faune. Mentionnons, à titre d'exemple, quelques-uns recensés dans ce journal: achigan (49, etc.); bécassine (888); bois blanc (49); canard de France (594); corneille (339); doré (102); gratia (116, etc.); lacaishe (428); liard (49); outarde (103); poires (403); prêle (752) et tremblière (294). On répertorie aussi des termes géo-toponymiques: brulés (569 et passim); chemin du large (66); embarras (744); hauteur des terres (77); islettes de bois (66); marais (40 et passim) et renversés (569 et passim) entre autres.

Les voyages dans ce territoire occasionnent aussi des emprunts, que l'on voyage par terre ou en canot, en été ou en hiver. Le vocabulaire ayant trait aux animaux, aux véhicules et au materiel fournit un certain nombre de termes dont apichimon "coussin" (270); ballotin "petite balle» (823); cassette «sorte de malle» (629 et passim); carriole (192 et passim); en canot (449); canotée (291 etc.); travail «brancards» (142); train «traineau» (170 et passim); voiture «traineau» (677). On trouve même chez cet auteur le verbe «to sault the rapids» (29) ainsi que son prétérit «We saulted...» (loc. cit., 31).

Le vocabulaire se rapportant à la traite des fourrures, commerce déjà bien établi au Détroit et aux pays plus loin à l'Ouest, s'avère (on s'en doutait déjà) une veine particulièrement riche dans la mine d'emprunts qu'était le français. Le personnel, les provisions, les réalités quotidiennes de la vie dans les régions limitrophes ainsi que les interactions avec les 
Amérindiens sont souvent représentés par des vocables français dont la plupart avait déjà figuré dans les écrits de Potier ou dans ceux de ses confrères. On trouve donc apalat «brochette de viande» (290, 410); bardache «homosexuel passif amérindien» (53 et passim); bourgeois "maître» (xvi, 49) et engagé «employé» (xvi); en cache "mise à l'abri, caché» (140 et passim); en derouine «à la recherche de pelleteries» (192 et passim); dépouille "graisse sous la peau du bison" (62 et passim); hivernant «qui passe l'hiver dans l'Ouest» (viii); paqueton "petit paquet de fourrures» (276); quenouilles "perches, supports aux coins d'une cheminée de terre» (579; v. Halford, Le français 291); taureau "peau de bison préparée comme récipient pour le pemmican» (173 et passim) et on en passe, car une liste complète des vocables français dans le journal de Henry dépasserait de loin les limites de la présente étude.

Enfin, les interchanges linguistiques dans les régions frontalières soulèvent deux questions d'étymologie qui méritent notre attention, bien que les premières attestations des vocables précèdent de quelque peu le début du dix-huitième siècle au sens large. Examinons d'abord celle de l'origine de l'anglais muskrat. Selon les autorités (Dictionary of Canadianisms 501b,-502a; Dictionary of Americanisms 1103b-1104a; SOED, I:1865c), la désignation aurait comme étymon l'algonquien musquash qui aurait été modifié au début du dix-septième siècle par étymologie populaire à l'actuel muskrat. Pour intéressante que soit cette explication, il me semble que les colons anglophones auraient pu arriver à la désignation de la même façon que leurs contemporains francophones: on nomme l'animal selon l'une de ses caractéristiques. En fait, on trouve dans la Relation du père La Jeune en 1635 (Thwaites VIII:44) l'explication du nom français de la bête: «On l'appelle Rat musqué pource qu'en effect une partie de son corps prise au Printemps sent le musc, en autres temps elle n'a point d'odeur". S'agit-il donc vraiment d'une étymologie populaire de la part des anglophones ou, par contre, d'une désignation métonymique, formée à l'anglaise avec le nom qualificatif devant le substantif, tout aussi logique que la dénomination française de l'animal qui est non seulement un membre de la famille des muridés comme le rat des champs, mais qui sent le musc tant au nez d'un Anglais qu'à celui d'un Français?

Par ailleurs, l'étymologie traditionellement proposée pour les Hurons, ce peuple amérindien, fidèles alliés des Français, nous paraît peu certaine. Certes, le mot huron est attesté en 1360 comme nom donné aux paysans révoltés, appelés aussi Jacques (Trésor de langue française IX:997a) et de 1380 au seizième siècle comme terme désignant un personnage grossier (ibid.; Thwaites IV:516a, ${ }^{*}$ hura) et ce sens est parfois invoqué pour expliquer l'attribution du nom au peuple, quelques-uns y voyant une insulte de la part des Européens. ${ }^{5}$ Cette hypothèse nous 
semble peu soutenable puisque les Français s'allient avec les Hurons dès les premiers moments de la colonie à Québec et on expliquerait avec difficulté l'attribution d'une dénomination insultante à un allié. Qui plus est, Champlain lui-même n'emploie le terme que dans son dernier livre en 1632. D'après Campeau, le fondateur de la Nouvelle-France appliquait à ce peuple en 1609-1611 soit le nom de leur chef, Ochateguin, soit la désignation Charioquois; en 1615-1616 il les désigne du nom de leur nation la plus nombreuse, Attigouantans (pour Attignaouantans) (26). Après cette date, Hurons devient courant et le frère Sagard s'en sert lors de sa visite au pays des Hurons en 1623.

Une autre explication de l'origine du nom figure dans la Relation du père Jérôme Lalement, missionnaire en Huronie en 1639 (Thwaites XVI:228-230). Selon cette source, une quarantaine d'années auparavant, lors de la première rencontre des indigènes et les Français, «quelque Matelot ou Soldat voyant pour la premiere fois cette sorte de barbare» ainsi que leur coiffure et «cette façon des cheueux luy semblant des hures, cela le porta à appeller ces barbares Hurons: \& c'est le nom qui depuis leur est demeuré». L'auteur ajoute que «Quelques-vns le rapportent à quelque autre semblable source, mais ce que nous en venons de dire semble le plus asseuré». Selon Campeau, éminent historien des Jésuites en Amérique, cette origine est "plausible» (26). Quant aux Hurons eux-mêmes, comme le note le père Lalement (loc. cit., 226), «Le nom general \& commun à ces quatre Nations, selon la langue du païs est (Ouendat)»; ce nom, Ouendat, ayant le sens de «dans la terre séparée», qui exprime, comme l'explique le père Campeau, «le sentiment qu'ont les Hurons d'être les hommes par excellence» (35). ${ }^{6}$

Néanmoins, il y a un élément encore plus pertinent pour notre étude, c'est le fait que, pour désigner différents groupes, la langue huronne disposait d'un suffixe populatif [-hrõnÕn], adjectif ethnique (Lagarde 402). Selon Pierrette-L. Lagarde, ce morphème, suffixé «au nom minimum ... indique soit un peuple soit un individu, selon qu'il est pris dans le sens général ou dans le sens particulier». Elle précise que le morphème «est traduit par: peuple, résidant, habitant. . . " Elle ajoute que le suffixe est "présent dans presque tous les noms de nations répertoriés» dans les Relations des Jésuites et qu'il "porte le pluriel $s$ français selon le contexte» (402). On trouve donc dans les Relations, à partir de 1635, des noms de groupes comme les Arendahronons "ceux de la roche», les Khionontatehronons "ceux des nombreuses collines», etc. (Lagarde 409-410), des désignations tant des Hurons et de leurs alliés que de leurs ennemis acharnés comme les Agniehronons «des groupes en canot» (414).

Il n'est pas exclu que les coiffures des Amérindiens et une attitude de supériorité de la part des Européens aient joué un rôle dans l'attribution 
du nom des Hurons. Il nous semble pourtant que, étant donnée l'importance politique et commerciale de l'alliance franco-ouendate et la fréquence du suffixe populatif [-hrÕnÕn], ainsi que la difficulté qu'aurait eu un Français d'articuler la série [-hrÕn-], c'est dans ce dernier que réside le véritable étymon de Huron.

Le but de notre conférence n'est pas d'examiner in extenso les échanges linguistiques entre les langues européennes et amérindiennes sur trois siècles et demi de contact: un tel examen dépasse de loin les limites du colloque. En fait, le champ est vaste. Une fouille sérieuse dans une optique linguistique des soixante-dix volumes des Relations reste à faire. Les journaux des explorateurs, tant anglophones que francophones, n'ont toujours pas été exploités à leur juste valeur linguistique. On n'a pas encore effectué une comparaison en profondeur des emprunts dans les différentes francophonies nord-américaines, depuis l'Acadie et le Québec jusqu'en Louisiane, en passant par le Détroit et la vallée du Mississipi, sans même parler de l'Ouest canadien. Une fouille des Dictionary of American English on Historical Principles et Dictionary of Canadian English on Historical Principles que Marcel Juneau a mentionnée voilà une vingtaine d'années n'a pas encore paru. La tâche d'établir les liens de parenté s'avère exigeante mais aussi très intéressante. Mentionnons, à titre d'exemple seulement, le journal d'Alexander Henry II: dans les cas d'étymons amérindiens, dans quels cas s'agit-il d'un emprunt direct et dans lesquels est-il question d'emprunt véhiculé par le français? Que dire des expressions figurées si importantes dans les cultures amérindiennes d'alors, ${ }^{7}$ et dont les traductions comme bury the hatchet, good/bad medicine et smoke the peace-pipe font toujours partie de l'anglais nordaméricain?

Tant que nous ne disposerons pas d'études de ce genre, les autorités débattront l'importance du rôle des langues amérindiennes dans l'évolution du français et de l'anglais dans le Nouveau Monde. Il est pourtant clair que même une étude si sommaire que la présente peut nous aider à évaluer plus justement les opinions rencontrées. Les faits linguistiques relevés dans la documentation indiquent que le jugement de Hall sur les interchanges entre les langues amérindiennes et le français (v. supra) n'a aucune base dans la réalité; la grande majorité des emprunts qui figurent dans les dictionnaires français modernes n'y sont pas parvenus par l'anglais. Ces faits infirme aussi l'hypothèse de Claude Poirier («Lexique») car les emprunts dépassent de loin le contexte limité des voyageurs et coureurs de bois.

Quant à la position de Marcel Juneau que «...l'apport du substrat amérindien, faible dans le québécois d'aujourd'hui, était jadis plus important», les relevés présentés ici tendent à la confirmer. Il est vrai que beaucoup des emprunts faisant partie d'anciennes formes du français et 
de l'anglais américains sont maintenant désuets mais la raison pour ces pertes se retrouve dans la société moderne: les réalités désignées (p. ex. apichimon, apola, mitasse etc.) ont disparu et avec elles leur désignation. On peut donc modifier quelque peu la conclusion de Juneau et dire avec raison que l'apport des langues amérindiennes au français de la Nouvelle-France ainsi que celui du français à l'anglais nord-americain étaient jadis bien plus importants qu'on ne l'imaginait.

\author{
PETER W. HALFORD \\ University of Windsor
}

\title{
Notes
}

1 Pierre Philippe Potier, jésuite, missionnaire, linguiste, premier curé de la première paroisse en Ontario, nous a légué un véritable trésor de renseignements sur la vie en Nouvelle-France à la veille de la Conquête. Parmi ses manuscrits qui nous sont parvenus, on trouve le seul lexique du français parlé de l'ancien régime (v. Halford, Le français), le seul recensement d'un village amérindien en Nouvelle-France (Toupin 176-268), la dernière grammaire huronne, ainsi que le dernier recueil de racines huronnes (v. Toupin et DBC IV: 692b-693b). Ses écrits touchent à tous les aspects de la société d'alors et nulle autre source nous communique si clairement le quotidien de la société frontalière où côtoyaient militaires et missionnaires, colons et coureurs de bois, artisans et Amérindiens. Par ailleurs, sa correspondance (v. Toupin 588-680) nous fournit un aperçu précieux de l'importance militaire et commerciale du Détroit du dix-huitième siècle.

2 Dictionary of Canadianisms consigne machecoti (454a) mais renvoie à matchcoat (468a-b). Dans l'article consacré à ce dernier vocable, on note l'attestation du début du dix-septième siècle dans l'ancienne Nouvelle-Angleterre (Dictionary of Americanisms 1035a) tout en signalant que la première attestation canadienne ne remonte qu'à 1768-1782, que la forme est matchee-coaty et que «... the early Canadian references [are] to a kind of woman's petticoat...», donc bien plus près du son et sens enregistrés par Potier.

3 Pour une explication possible de l'origine, souvent questionnée, du terme panis, v. Halford, Le français 225-226.

4 Cf. les relevés de Potier dans Halford, «Le vocabulaire».

5 Cf., entre autres, Heidenreich 21.

6 Il ne nous manque pas d'exemples de peuples américains qui s'appellent tout simplement «le peuple», «le véritable peuple», «les hommes» ou «les gens» comme le font les Inuit, les Inupiaq et les Ilini.

7 Déjà en 1636, Brébeuf avertit ses confrères missionnaires éventuels «Les metaphores sont grandement en vsage parmy ces Peuples; si vous ne vous y faites, vous n'entendez rien dans leurs conseils, où ils ne parlent quasi que par metaphores» (Thwaites X:218). 


\section{BIBLIOGRAPHIE SOMMAIRE}

\section{Manuscrits}

Almazan, Vincent, Les Canadiens français du Détroit, leur parler. Manuscrit dactylographié. Belle-Rivière, 1977. Dans la collection du TLFQ, Université Laval.

Bénéteau, Marcel. Achaïsmes et régionalismes dans un corpus de chansons folkloriques $d u$ Détroit. Mémoire de premier cycle. University of Windsor, Windsor, 1992.

Blais, Suzelle, éd. Néologie canadienne, ou Dictionnaire des mots créés en Canada et maintenant en vogue; des mots dont la prononciation et l'ortographe sont différentes de la prononciation et ortographe françoises, quoique employés dans une acception semblable ou contraire; et des mots étrangers qui se sont glissés dans notre langue. Jacques Viger. Édition commentée des manuscrits de Viger. Manuscrit dactylographié. Québec, 1982. Dans la collection du TLFQ, Université Laval.

Johnson, Neil H. The Sugar Bush Speech of the Detroit French Dialect. Mémoire de maîtrise. Wayne State University, Detroit, 1951.

\section{Imprimés}

Askin, John. The John Askin Papers. Ed. Milo M. Quaife. 2 vols. Detroit, Detroit Library Commission, 1928, 1931.

Bonnefons, J.-C. Voyage au Canada dans l'Amérique Septentrionale fait depuis l'an 1751 à l'an 1761. Ed. H. K. Casgrain. Québec: Brousseau 1887; Paris, 1978.

Campeau, Lucien. La mission des Jésuites aux Hurons 1634-1650. Montréal: Éditions Bellarmins; Roma: Intitutum Historicum S.I., 1987.

Clapin, Sylva. Dictionnaire canadien-français. 1894. Québec: PUL, 1974.

Dictionary of Americanisms on Historical Principles. Éd. Mitford Macleod. Chicago, Londres, Toronto: U of Chicago P, 1951.

Dictionary of Canadianisms on Historical Principles. Lexicographical Centre for Canadian English. Toronto: Gage, 1967.

Dictionnaire biographique du Canada/Dictionary of Canadian Biography. Quebec et Toronto, 1966-. (DBC)

Dictionnaire du français plus: à l'usage des francophones d'Amérique. Montréal: Centre éducatif et culturel, 1988.

Dionne, Narcisse-Eutrope. Le Parler populaire des Canadiens français, ou Lexique des canadianismes. 1909. Québec: PUL, 1974.

Dulong, Gaston. Dictionnaire des canadianismes. Montréal: Larousse, 1989.

Dunn, Oscar. Glossaire franco-canadien. 1880. Québec: PUL, 1976.

Glossaire du parler français au Canada. La Société du parler français au Canada. 1930. Québec: PUL, 1968. 
Halford, Peter W. Le français des Canadiens à la veille de la Conquête: témoignage de Pierre Philippe Potier, s.j. Ottawa: Presses de l'Université d'Ottawa, 1994.

. "Le Vocabulaire de la frontière au XVIII ${ }^{\mathrm{e}}$ siècle: quelques relevés du père Pierre Philippe Potier S.J.» Le français des Amériques. Robert Fournier et Henri Wittmann. Trois-Rivières: Presses universitaires de Trois-Rivières, 1995. 231-246.

Hall, Robert A. External History of the Romance Languages. 2 vols. Amsterdam, Oxford, New York: American Elzevier, 1974, 1976.

Hanzeli, Victor Egon. Missionary Linguistics in New France. A Study of Seventeenth-and Eighteenth-Century Descriptions of American Indian Languages. The Hague, Paris: Mouton, 1969.

Heidenreich, Conrad. Huronia, a History and Geography of the Huron Indians, 1600-1650. Toronto: McLelland and Stewart, 1971.

Henry, Alexander (1739-1824). Travels and Adventures in Canada and the Indian Territories between the Years 1760 and 1776. 1809. Facsimilé. Ann Arbor: University Microfilms, 1966.

Henry, Alexander (1765-1814). New Light on the early history of the Greater Northwest. The manuscript journals of Alexander Henry. Éd. Elliott Coues. 1897. Minneapolis: Ross and Haines, 1965.

Hull, Alexander. The Franco-Canadian dialect of Windsor, Ontario: a preliminary study. Thèse de doctorat. University of Washington, 1955.

Juneau, Marcel. Problèmes de lexicologie québécoise. Prolégomènes à un Trésor de la langue française au Québec. Québec: PUL, 1977.

Lagarde, Pierrette-L. «La formation des noms de peuples et de bourgades en Huron». Campeau, Mission, Appendice III. 375-450.

New Shorter Oxford English Dictionary. 2 vols. Oxford: Clarendon Press, 1993. (SOED)

Poirier, Claude. «Le lexique québécois: son évolution, ses composantes». Culture populaire et littérature au Québec. Stanford French and Italian Studies. Vol. 19. Ed. René Bouchard. Saratoga: Anma Libri, 1980. 43-80.

Thwaites, Reuben Gold, éd. The Jesuit Relations and Allied Documents. Travels and Explorations of the Jesuit Missionaries in New France, 1610-1791, 1896-1901. 73 vol. New York: Pageant Book Company, 1959.

Toupin, Robert. Les Écrits de Pierre Potier. Ottawa: Presses de l'université d'Ottawa, 1996.

Trésor de la langue française. Publié sous la direction de Paul Imbs. Paris: Centre nationale de la recherche scientifique. 1971-.

Wartburg, Walter von. Französisches Etymologisches Wörterbuch. Bonn-Leipzig: Bâle, 1922- 